\title{
I-131 Postablation SPECT/CT Predicts Relapse of Papillary Thyroid Carcinoma more Accurately than Whole Body Scan
}

\author{
JULIA V. MALAMITSI ${ }^{1}$, JOHN T. KOUTSIKOS ${ }^{2}$, STAMATIA I. GIOURGOULI ${ }^{1}$, SOPHIA F. ZACHAKI ${ }^{3}$, \\ THEODOROS A. PIPIKOS ${ }^{4}$, FANI J. VLACHOU ${ }^{4}$ and VASSILIOS K. PRASSOPOULOS ${ }^{4}$ \\ ${ }^{1}$ Department of Medical Physics, Medical School, National and Kapodistrian University of Athens, Athens, Greece; \\ ${ }^{2}$ Department of Nuclear Medicine, Army Share Fund Hospital (417 NIMTS), \\ Athens Greece and Department of Nuclear Medicine, Henry Dunant Hospital Centre, Athens, Greece; \\ ${ }^{3}$ Laboratory of Health Physics, Radiobiology and Cytogenetics, \\ National Centre for Scientific Research (NCSR) "Demokritos", Athens, Greece; \\ ${ }^{4}$ Nuclear Medicine Department, Hygeia Hospital, Maroussi, Greece
}

\begin{abstract}
Background/Aim: The aim of the study was to prospectively compare I-131 postablation Whole Body scan (WBS) and Single Photon Emission Computerized Tomography/Computerized Tomography (SPECT/CT) scan on thyroid cancer patients. Patients and Methods: Overall, 58 patients with papillary thyroid carcinoma were submitted to total thyroidectomy and I-131 remnant ablation. Postablation WBS and SPECT/CT scans performed on the same day were compared. Results of SPECT/CT were confirmed by neck and upper mediastinum ultrasound scan and on specific cases by a fully diagnostic CT scan, other tests and definitive histology acting as the gold standard. A total of 36/58 patients were followed-up for 5 years to detect relapse. Results: Mac Nemar Chi square and Fisher's exact tests disclosed statistically significant differences between WBS and SPECT/CT scan, concerning cervical lymphadenopathy detection $(p=0.031)$ and relapse prediction by NM stage $(p=0.033)$, respectively; SPECT/CT was more accurate in both comparisons. Conclusion: In papillary thyroid carcinoma I-131 post-ablation SPECT/CT scan detects cervical lymphadenopathy and predicts relapse by NM stage more accurately than WBS.
\end{abstract}

This article is freely accessible online.

Correspondence to: Julia V. Malamitsi, Department of Medical Physics, Medical School National and Kapodistrian University of Athens, Greece, 75 Mikras Asias str., Athens 115 27, Greece. Tel: +30 2107462368, +30 2107462375, +30 6947528447, e-mail: j.malamitsi@yahoo.gr; imalamits@med.uoa.gr

Key Words: Papillary thyroid carcinoma, I-131 postablation scan, whole body scan, SPECT/CT.
I-131 post-ablation Whole Body scan (WBS) in patients with a well differentiated thyroid carcinoma (DTC) delivers more information than the pre-ablation diagnostic scan due to the high I-131 ablative dose delivered, and therefore is capable of altering stage and therapeutic management of patients (1, 2). However, planar WBS with I-131 in patients with DTC offers poor imaging data. On the contrary, a SPECT/CT scan obtains three dimensional images and offers accurate anatomometabolic information (3). There is growing evidence that SPECT/CT, especially with a dedicated hybrid device, contributes to a higher diagnostic accuracy by detecting infiltrated lymph nodes and distant metastases from DTC, accurately localizing the metastatic foci and differentiating physiological from pathological uptake of the radiopharmaceutical (3-6). This information contributes to a more accurate TNM staging and consequently to a better risk stratification of DTC patients and therapeutic management $(3,7)$. Therefore SPECT/CT has an increasing use in the detection of metastatic disease in DTC.

The aim of this study was to compare I-131 post-ablation SPECT/CT scan with planar I-131 post-ablation Whole Body scan (WBS) in restaging of patients with DTC after total thyroidectomy and ablation therapy, as well as in predicting relapse within a long period of follow-up. We planned a prospective study with a dedicated hybrid SPECT/CT device.

\section{Patients and Methods}

Fifty-eight patients with DTC who had undergone total thyroidectomy followed by I-131 remnant ablation, were enrolled in the study between December 2011 and October 2018. All 58 patients, 25 men and 33 women aged 30 to 82 years, were diagnosed with DTC of the papillary type. After total thyroidectomy a diagnostic I-131 uptake measurement with $2.22 \mathrm{MBq}(60 \mu \mathrm{Ci})$ was performed, in order to appreciate remnant thyroid tissue presence. Thyroglobulin ( $\mathrm{Tg}$ ) levels and antithyroglobulin 
antibodies (TgAb) were measured. I-131 ablative doses ranged from 2775 to $5550 \mathrm{MBqs}(75-150 \mathrm{mCi})$. Five to seven days after the ablative dose a post therapy WBS was acquired using a SOPHA dual DST XLI Nuclear Gamma Camera. A SPECT/CT scan of the neck and thorax was performed on a Philips BrightView XCT device on the same day. Informed consent was obtained from all the enrolled patients and the protocol was approved by the Ethical Committee of Hygeia Hospital, adhering to the principles of the Declaration of Helsinki.

A subsequent neck and upper mediastinum U/S scan was performed on all patients, in order to evaluate thyroid and thyroglossal cyst remnants and abnormal cervical lymph nodes $(8$, 9). When distant findings were present on WBS of these 58 patients, an extra SPECT/CT study was performed to clarify these findings in the respective bed position. On dubious cases a fully diagnostic CT scan was planned, and occasionally another test. WBS and SPECT/CT scans were read separately and in comparison, by two experienced Nuclear Medicine physicians. As gold standard, we used surgery with definitive histology, a neck and upper mediastinum U/S on occasions of a fully diagnostic CT scan, as well as close clinical and radiological monitoring along with changes in Tg. The patients are followed up to this date. On thirtysix patients who completed a 5-year follow-up, the relapse rate was assessed.

A Mc Nemar chi square statistical test was used to determine whether I-131 planar WBS and SPECT/CT distributions had significant differences. Cohen's $\mathrm{K}$ statistics was calculated in order to assess the agreement between the two methods. Fisher's exact test was used to assess differences in proportion of patients relapsing within five years by NM stage, as determined by WBS and SPECT/CT. All $p$-values reported were 2 -sided. A $p$-value $<0.05$ was considered significant. Statistical analysis was performed using SPSS (Statistical Package for the Social Sciences) version 20 software.

\section{Results}

Fifty-eight patients with papillary thyroid carcinoma underwent a post-ablation I-WBS and a SPECT/CT scan of the neck and thorax, respectively. Ten out of them required an additional SPECT/CT scan on another bed position, because of indeterminate findings in distant areas on the WBS. All patients had a pre-ablation TSH value above 30 $\mathrm{mUI} / \mathrm{l}$. TSH, Tg and postoperative uptake values are presented in Table I. Antithyroglobulin antibodies were present in four patients.

We used a focus-based and an NM stage-based patient analysis on our data. All radioactive foci on WBS and SPECT/CT were grouped into six categories, i.e., presence/absence of thyroid remnant, thyroglossal cyst, thymus, cervical lymph nodes, mediastinal foci/nodes and distant metastases (Table II) (Figures 1a and b, 2a and b, 3a and b). Neck and upper mediastinum U/S, additional SPECT/CT views on other than neck and lung areas as well as a fully diagnostic CT scan helped in further characterizing foci as benign or malignant. In particular in four cases, three with lung metastases (Figure $2 \mathrm{a}$ and $\mathrm{b}$ ) and one with an
Table I. Sample characteristics - TSH, Tg and post-op uptake (\%) $(N=58)$.

\begin{tabular}{lcc}
\hline & Median (IQR) & Range \\
\hline $\mathrm{TSH}(\mathrm{mUI} / \mathrm{l})$ & $83(65,112)$ & $30.9,>200$ \\
$\mathrm{Tg}(\mathrm{ng} / \mathrm{ml})$ & $3(0,10)$ & $<0.2,47.1$ \\
Post-op uptake $(\%)$ & $3(1,5)$ & $0.5,15.4$ \\
\hline
\end{tabular}

infiltrated pericolic lymph node (Figure 4), a fully diagnostic CT scan confirmed SPECT/CT findings. On a patient with abdominal foci and presumed bowel activity on SPECT/CT, a colonoscopy confirmed SPECT/CT results. Four patients staged as N0M0 on WBS and as N1M0 on SPECT/CT were submitted to lateral compartment cervical lymphadenectomy. Definitive histology showed infiltrated lymph nodes.

Concerning the focus-based analysis, on the total number of findings, WBS gave 27 false positive (7.76\%) and 23 false negative $(6.61 \%)$ results (Table II).

After performing SPECT/CT, several changes were induced in $\mathrm{N}$ and $\mathrm{M}$ staging in the framework of TNM classification, as compared with the initial stage estimation which comprised histopathology and WBS findings. On cross tabulation of NM stage through WBS and SPECT/CT only 24 patients with N0M0 stage and 6 patients with N1M0 stage were classified correctly in the same stage by both methods (Table III). Considering N stage, there were 14 cases of downstaging on SPECT/CT from N1 to N0 and 8 cases of upstaging from N0 to N1. Considering $\mathrm{M}$ stage, there were two cases of downstaging from M1 to M0 and 5 cases of upstaging from M0 to M1. Cohen's $\mathrm{k}$ was 0.053 and not statistically significant $(p=0.307)$, which signified that the degree of agreement between the two methods was very low. The accuracy of WBS in determining NM stage was low $(51.72 \%)$, while that of SPECT/CT $100 \%$ respectively.

Mc Nemar chi square statistical analysis of our data revealed a statistically significant difference concerning the results of infiltrated cervical lymph nodes between the two methods $(p=0.031)$. This means that SPECT/CT could detect infiltrated cervical lymph nodes more accurately than WBS, by decreasing false negative and false positive findings. No statistically significant difference between WBS and SPECT/CT distributions $(p>0.05)$ concerning detection of thyroid remnant, thyroglossal cyst, thymus, mediastinal foci/lymph nodes and distant metastases was noticed.

Thirty-six out of fifty-eight patients were followed up for 5 years and the relapse rate by NM stage on WBS and SPECT/CT was assessed (Table IV). In total, four cases of relapse were observed. These cases of relapse were attributed to NM stages as classified by WBS and SPECT/CT. A $4.3 \%$ of patients in NOMO stage by SPECT/CT presented with 
Table II. Focus based analysis on WBS and SPECT/CT results. False-positive and false-negative results of WBS. All figures represent N (\%).

\begin{tabular}{lcccc}
\hline Focus & Both + & Both - & FP & FN \\
\hline Thyroid remnant & $50(86.21 \%)$ & $5(8.62 \%)$ & $2(3.45 \%)$ & $1(1.72 \%)$ \\
Thyroglossal cyst & $11(18.97 \%)$ & $36(62.07 \%)$ & $6(10.34 \%)$ & $5(8.62 \%)$ \\
Thymus & $2(3.45 \%)$ & $53(91.38 \%)$ & $1(1.72 \%)$ & $2(3.45 \%)$ \\
Cervical lymph nodes & $6(10.34 \%)$ & $33(56.90 \%)$ & $14(24.14 \%)$ & $5(8.62 \%)$ \\
Mediastinal foci/Nodes & $1(1.72 \%)$ & $50(86.21 \%)$ & $2(3.45 \%)$ & $5(8.62 \%)$ \\
Distant metastases & $0(0.00 \%)$ & $51(87.93 \%)$ & $2(3.45 \%)$ & $5(8.62 \%)$ \\
\hline All foci & $70(20.11 \%)$ & $228(65.52 \%)$ & $27(7.76 \%)$ & $23(6.61 \%)$ \\
\hline
\end{tabular}

Table III. Cross tabulation of N, M Stage by SPECT/CT vs. WBS (N=58). Cohen's $\mathrm{k}$ statistics assesses agreement between the two methods.

\begin{tabular}{lccccc}
\hline & \multicolumn{5}{c}{ N, M Stage - WBS } \\
\cline { 2 - 5 } & N0M0 & N1M0 & N0M1 & Overall \\
N $(\%)$ & N $(\%)$ & & $p$-Value (Cohen's K) \\
\hline N, M Stage - SPECT/CT & $24(70.6)$ & $14(63.6)$ & $2(100.0)$ & $40(69.0)$ & $0.307(0.053)$ \\
N0M0 & $7(20.6)$ & $6(27.3)$ & $0(0.0)$ & $13(22.4)$ \\
N1M0 & $2(5.9)$ & $0(0.0)$ & $0(0.0)$ & $2(3.4)$ & $3(5.2)$ \\
N0M1 & $1(2.9)$ & $2(9.1)$ & $0(0.0)$ & $58(100.0)$ \\
N1M1 & $34(58.6)$ & $22(37.9)$ & $2(3.45)$ & \\
\hline Total & &
\end{tabular}

relapse, while for patients with a higher than N0M0 stage by SPECT/CT, the proportion was $23.1 \%$. In particular, in patient group NOM0, one out of 23 patients had a relapse, in the N1M0 group one out of 8 and in the N1M1 group two out of three (Table IV).

Fisher's exact test pointed the statistical differences in the proportion of patients relapsing within 5 years by NM stage as determined through WBS and SPECT/CT. There was a statistically significant difference in relapse proportion by NM stage when assessed with SPECT/CT ( $p=0.033)$, while with WBS this difference was not significant $(p=0.209)$ (Table IV).

\section{Discussion}

The established way of staging a DTC postoperatively is by planar I-131 WBS together with serum thyroglobulin measurements (10). This helps evaluate thyroid remnant and presence of regional and distant metastases. Post-ablation WBS with thyroglobulin levels are also used for restaging and follow-up $(11,12)$. However, SPECT/low dose CT discloses more foci in comparison with planar WBS and U/S and offers a more accurate localization of the foci. SPECT/CT allows a differentiation between physiological uptake of the radiopharmaceutical and malignant uptake, leading to a more effective therapy $(3,13)$. In comparison with WBS, SPECT/CT improves diagnostic performance because it reveals more pathological lesions, reduces false positive and equivocal findings, alters nodal stage and risk stratification of the patients, and has therefore a significant impact on early risk stratification, therapy, management and prognosis of DTC $(3,5,7,14-24)$. In addition, SPECT/CT can clarify false positive uptake of I-131 due to sodium iodide symporter expression by tissues and consequent trapping to inflammation/infection, and to increased perfusion etc. $(25,26)$. In cases with negative I-131 WBS and elevated serum thyroglobulin FDG-PET could detect all patients with metastatic cervical lymph nodes, while technetium-99m tetrofosmin SPECT as well as technetium $99 \mathrm{~m}$ methoxyisobutylisonitrile SPECT could detect only a proportion of the affected patients $(27,28)$.

Due to all the above mentioned reasons, we chose to perform a SPECT/CT scan on all our studied patients. Results of SPECT/CT were confirmed by neck and upper mediastinum U/S scan and on specific cases by a fully diagnostic CT scan. In one patient an additional colonoscopy confirmed the presence of bowel activity as pointed by 


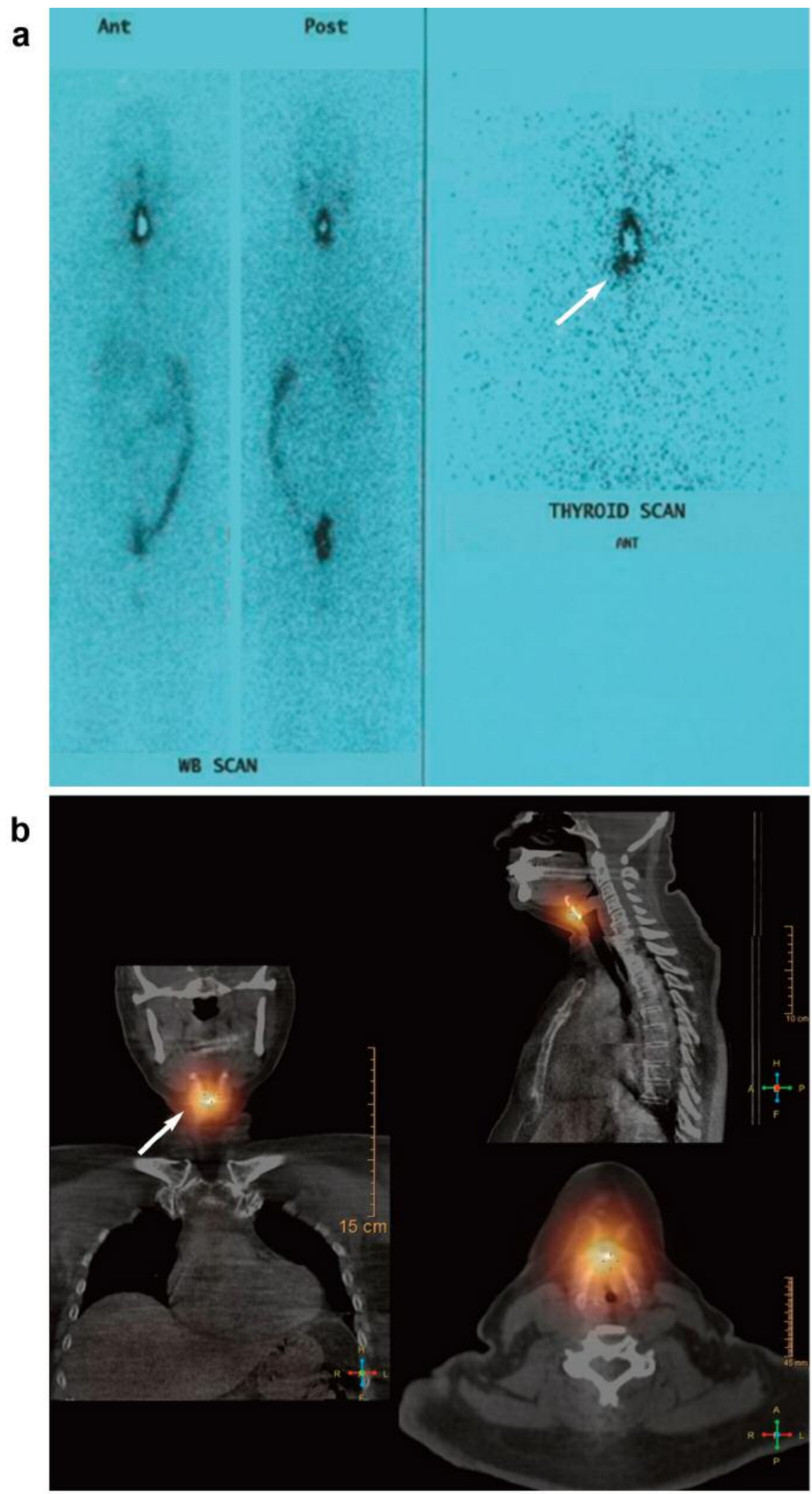

Figure 1. Thyroglossal cyst remnant on SPECT/CT. a: Increased radioactive uptake is seen on WBS in an area considered as thyroid remnant. b: On SPECT/CT the same area is a thyroglossal cyst remnant (white arrow). 
a

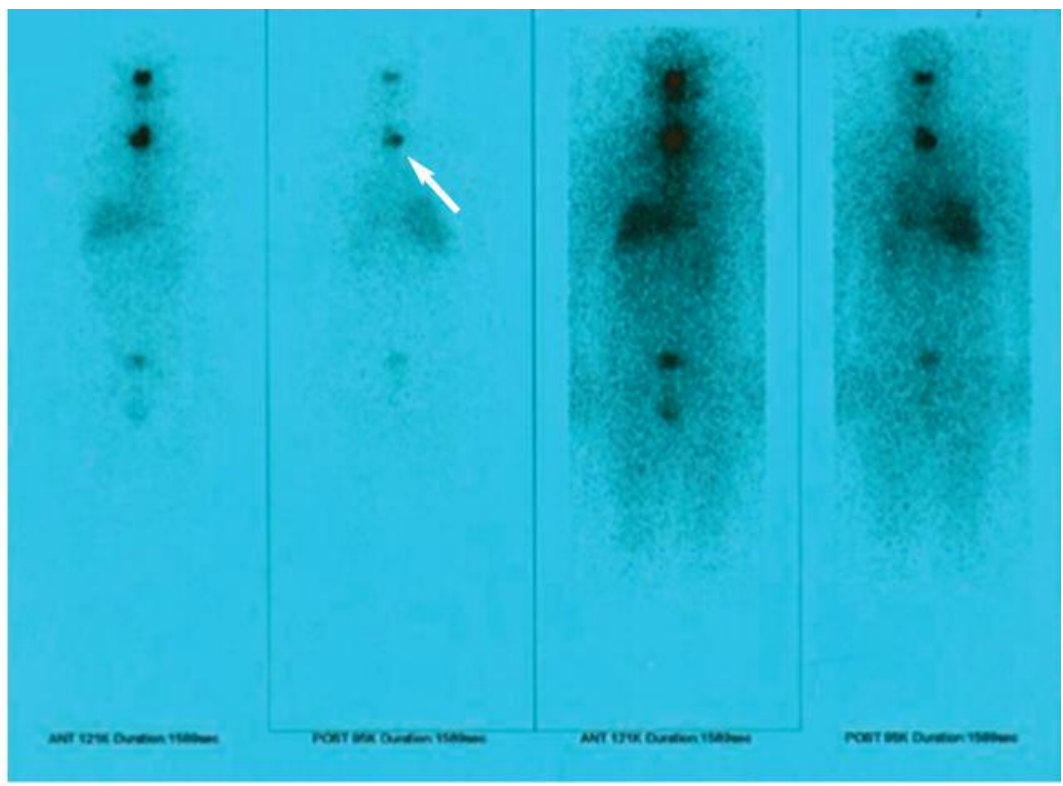

b

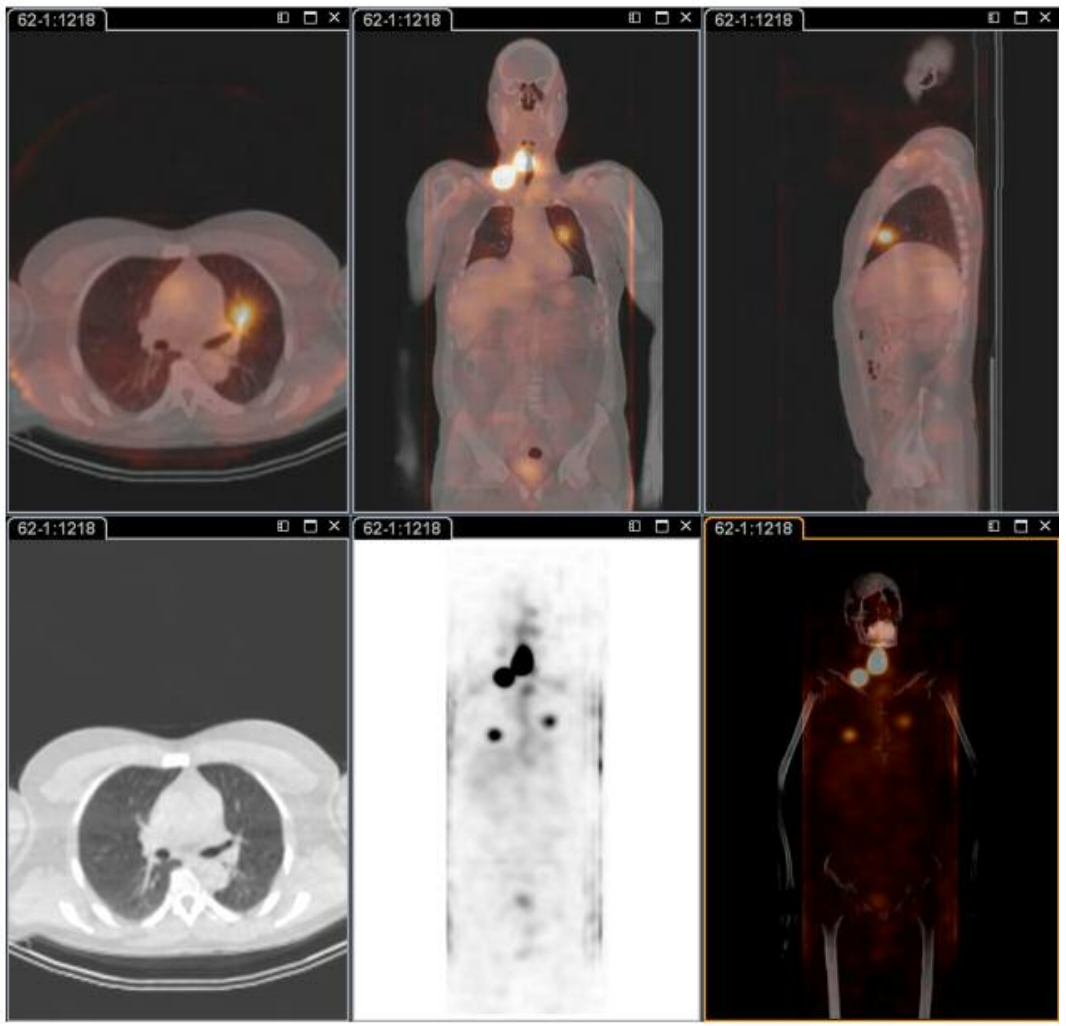

Figure 2. False-negative WBS. a: WBS showing high radioactive uptake corresponding to the thyroid remnant (white arrow). No pathological uptake is seen elsewhere. $b$ : SPECT/CT on the same patient shows areas of high radioactive uptake corresponding to the thyroid remnant, a right regional cervical lymph node and two lung foci, one right and one left.

SPECT/CT. In patients who received a lateral compartment lymphadenectomy, histology confirmed SPECT/CT results.

SPECT/CT proved particularly helpful in cases considered as indeterminate on WBS, i.e. cases with significant thyroid remnant and doubtful presence of cervical lymph nodes, increased $\mathrm{Tg}$ which could not be justified by the existing thyroid remnant, suspicion of presence of a thyroglossal cyst and lastly in cases with 
a
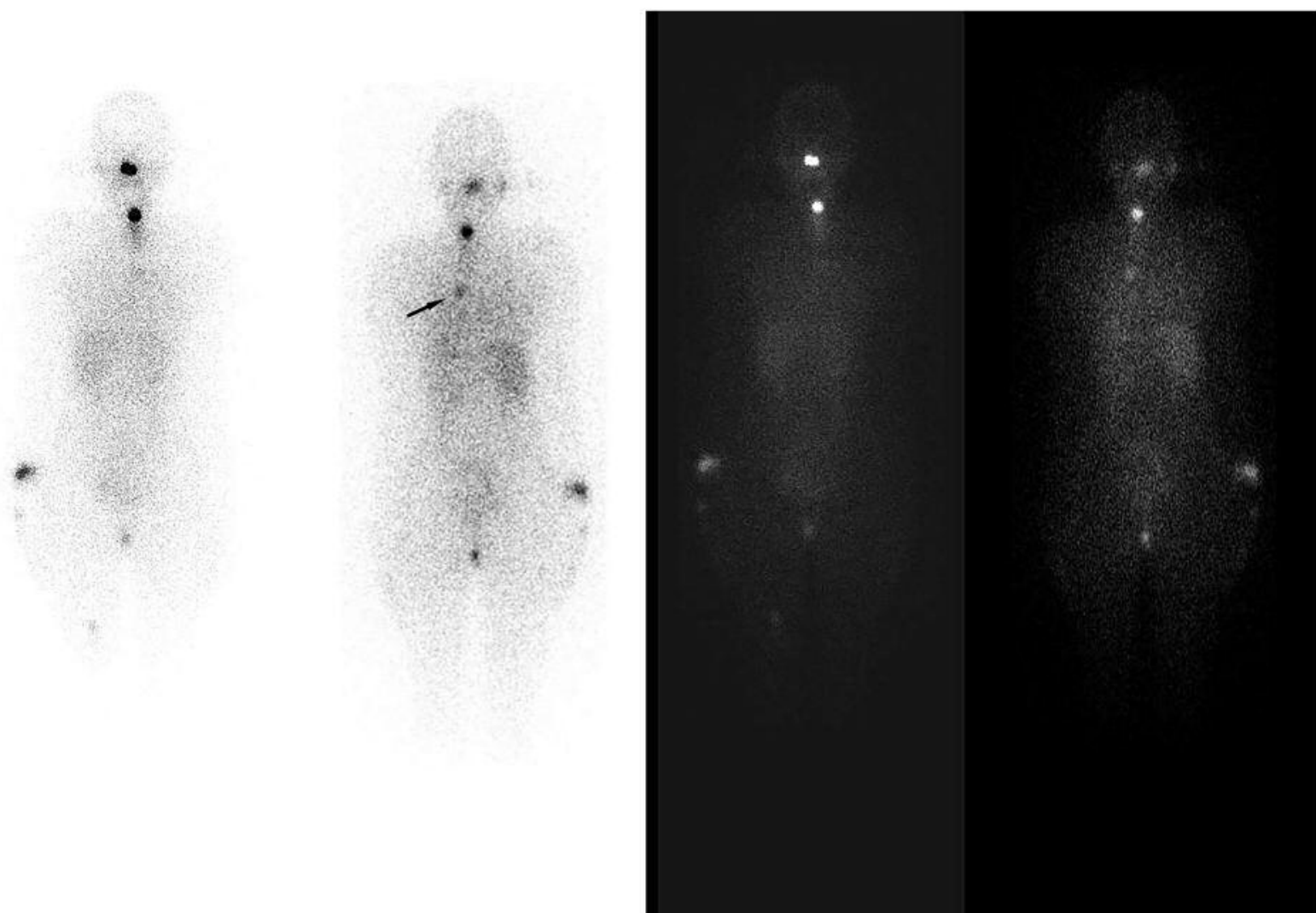

b
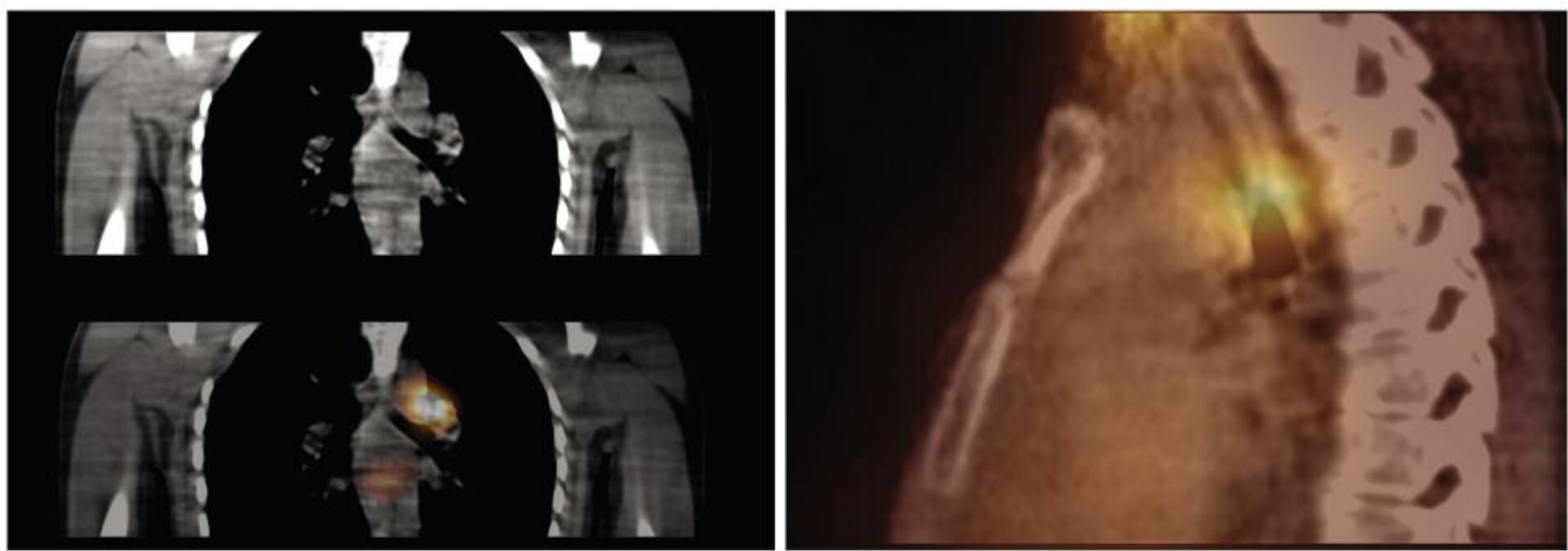

Figure 3. False-positive WBS. a: WBS demonstrates an area of high radioactive uptake in the upper mediastinum to the left of the midline (black arrow). $b$ : SPECT/CT of the thorax on the same patient shows that the high uptake area corresponds to oesophageal activity.

dubious uptake in the abdominal area and other distant areas not attributable to physiologic or pathologic activity with certainty.

In our study, by comparing WBS with SPECT/CT in the same patients we concluded that SPECT/CT is significantly more accurate in demonstrating infiltrated regional cervical lymph nodes, by reducing false-positive and false-negative results provided by WBS. On the other hand, SPECT/CT disclosed infiltrated mediastinal lymph nodes and distant metastases, thus reducing false negatives, but not to a statistically significant degree. In the cervical lymph node work up, hybrid imaging clarified the poor imaging results of planar WBS. Due to low dose CT the anatomic information on the exact number of infiltrated cervical 

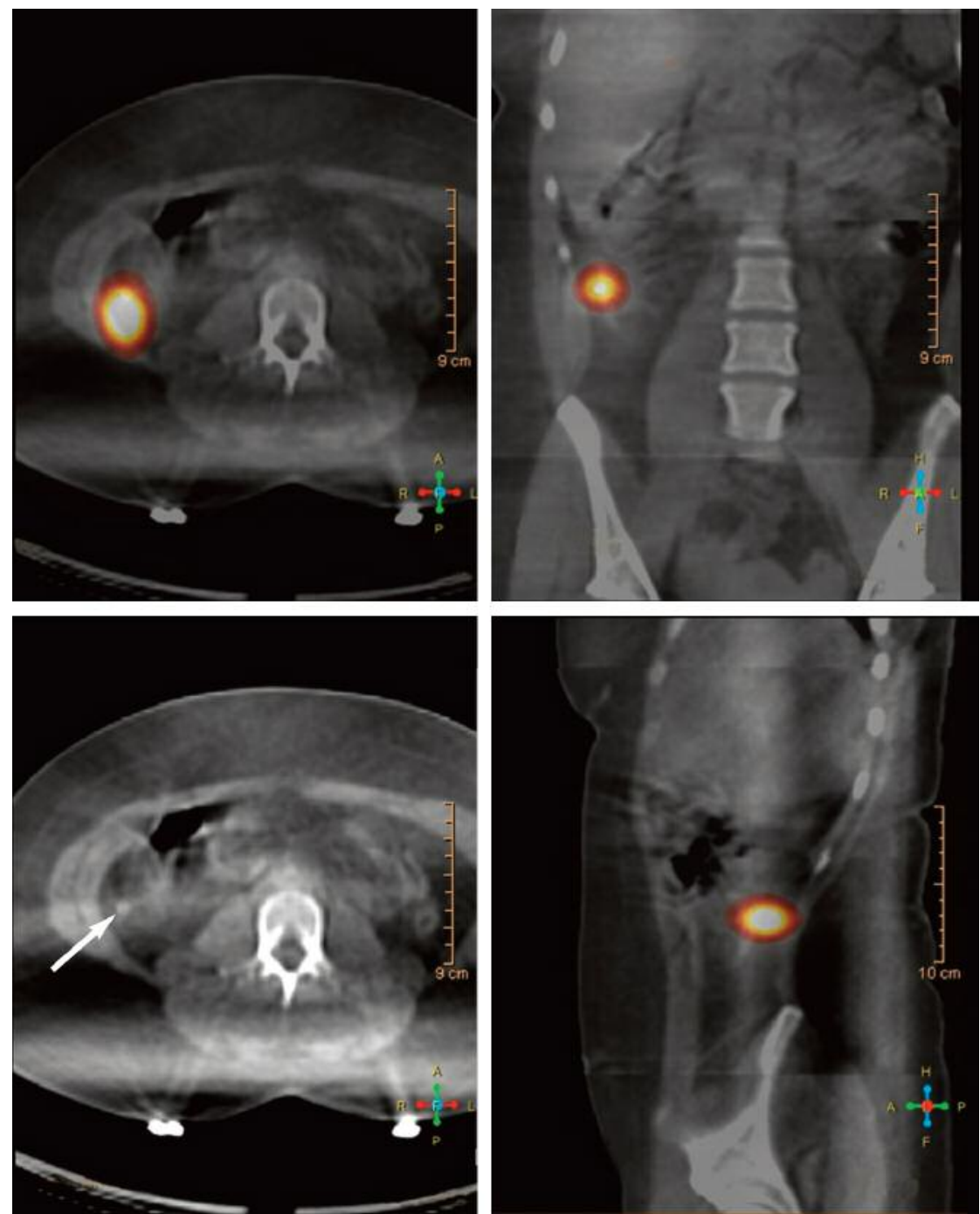

Figure 4. High radioactive uptake is seen on SPECT/CT corresponding to a right paracolic lymph node (white arrow on CT). The same area of increased uptake on WBS was considered physiological bowel uptake.

lymph nodes was limited, however, on some occasions we did not wish to increase the radiation dose absorbed by the patients with an additional fully diagnostic CT. On WBS the streak artifact from a thyroid remnant may cover up malignant lesions in the mediastinum and ribs and since differentiation between skeletal and soft tissue structures by low dose CT is satisfactory, SPECT/CT was proven accurate in disclosing a rib bony metastasis. In addition, SPECT/CT could demonstrate uptake on metallic dental restorations mostly in the mandibular bone and in the left maxillary sinus due to infection, leading to downstaging of the patient $(6,29,30)$.

Concerning radioactive contamination, SPECT/CT could differentiate contamination from malignant bone involvement which is difficult on planar WBS, since it requires washing of
Table IV. Proportion of patients relapsing within 5 years by $N$, M Stage $(N=36)$.

\begin{tabular}{|c|c|c|c|c|}
\hline & \multicolumn{2}{|c|}{ Relapse } & \multirow[b]{2}{*}{$\begin{array}{l}\text { Overall } \\
\mathrm{N}(\%)\end{array}$} & \multirow[b]{2}{*}{$p$-Value } \\
\hline & $\begin{array}{c}\text { No } \\
\text { N (\%) }\end{array}$ & $\begin{array}{c}\text { Yes } \\
\text { N (\%) }\end{array}$ & & \\
\hline N, M Stage- WBS & & & & 0.209 \\
\hline NOM0 & $21(95.5)$ & $1(4.5)$ & $22(100.0)$ & \\
\hline N1M0 & $9(75.0)$ & $3(25.0)$ & $12(100.0)$ & \\
\hline N0M1 & $2(100.0)$ & $0(0.0)$ & $2(100.0)$ & \\
\hline $\mathrm{N}$, M Stage - SPECT/CT & & & & 0.033 \\
\hline NOM0 & $22(95.7)$ & $1(4.3)$ & $23(100.0)$ & \\
\hline N1M0 & $7(87.5)$ & $1(12.5)$ & $8(100.0)$ & \\
\hline N0M1 & $2(100.0)$ & $0(0.0)$ & $2(100.0)$ & \\
\hline N1M1 & $1(33.3)$ & $2(66.7)$ & $3(100.0)$ & \\
\hline
\end{tabular}


the respective area, additional projections and delayed acquisitions. Finally, SPECT/CT helped clarify the presence of thyroid remnant, thyroglossal cyst and thymus (31).

Considering the statistical analysis performed, a statistically significant difference was found between the two methods only in the results concerning infiltrated cervical lymph nodes $(p=0.031)$. In addition, staging performed by SPECT/CT was significantly associated with a probability of relapse $(p=0.033)$, whereas this was not the case with WBS results $(p=0.209)$.

The advantages of our study are the following: We planned a prospective study on 58 DTC patients with a planar WBS and a SPECT/CT scan on a dedicated hybrid device and compared the results. To our knowledge this is the first Greek study concerning post-ablation SPECT/CT in papillary thyroid carcinoma. Our study was focus-based so that we could sum up all false negative and false positive findings, but it was also NM stage-based, especially in terms of follow-up (13).

In conclusion the results of our study showed that in patients with DTC, post-ablation I-131 SPECT/CT was superior to WBS in determining cervical lymphadenopathy and in predicting relapse by NM stage classification to a statistically significant degree.

\section{Conflicts of Interest}

The Authors of this study declare no conflicts of interest.

\section{Authors' Contributions}

Doctors J. Koutsikos and S. Giourgouli recruited the 58 patients of the study. They performed I-131 diagnostic uptake measurements, administered the I-131 therapeutic dose and performed the WBS. They follow up patients till now; Doctors V. Prassopoulos, Th. Pipikos and F. Vlachou performed SPECT/CT studies on all 58 patients. Doctors Prassopoulos and Koutsikos read all WBS and SPECT/CT scans separately and in comparison; Dr J. Malamitsi processed the data and wrote the manuscript; Dr S. Zachaki performed the statistical analysis of the data.

\section{References}

1 Fatourechi V, Hay ID, Mullan BP, Wiseman GA, EghbaliFatourechi GZ, Thorson LM and Gorman CA: Are posttherapy radioiodine scans informative and do they influence subsequent therapy of patients with differentiated thyroid cancer? Thyroid 10: 573-577, 2000. PMID: 10958309. DOI: 10.1089/thy.2000.10.573

2 Souza Rosario PW, Barroso AL, Rezende LL, Padrão EL, Fagundes TA, Penna GC and Purisch S: Post I-131 therapy scanning in patients with thyroid carcinoma metastases: an unnecessary cost or a relevant contribution? Clin Nucl Med 29: 795-798, 2004. PMID: 15545881.

3 Spanu A, Solinas ME, Chessa F, Sanna D, Nuvoli S and Madeddu G: 131I SPECT/CT in the follow-up of differentiated thyroid carcinoma: incremental value versus planar imaging. J Nucl Med
50: 184-190, 2009. PMID: 19164225. DOI: 10.2967/jnumed. 108.056572

4 Mustafa M, Kuwert T, Weber K, Knesewitsch P, Negele T, Haug A, Linke R, Bartenstein P and Schmidt D: Regional lymph node involvement in $\mathrm{T} 1$ papillary thyroid carcinoma: a bicentric prospective SPECT/CT study. Eur J Nucl Med Mol Imaging 37: 1462-1466, 2010. PMID: 20358197. DOI: 10.1007/s00259-0101408-2

5 Maruoka Y, Abe K, Baba S, Isoda T, Sawamoto H, Tanabe Y, Sasaki $M$ and Honda $H$ : Incremental diagnostic value of SPECT/CT with 131I scintigraphy after radioiodine therapy in patients with well-differentiated thyroid carcinoma. Radiology 265 : 902-909, 2012. PMID: 23012466. DOI: 10.1148/radiol.12112108

6 Shapiro B, Rufini V, Jarwan A, Geatti O, Kearfott KJ, Fig LM, Kirkwood ID and Gross MD: False-Positive Radioiodine Scans in Thyroid Cancer. In: Thyroid Cancer, A Comprehensive Guide to Clinical Management, Leonard Wartofsky, Douglas Van Nostrand (eds). Springer, pp. 185-204, 2016.

7 Schmidt D, Szikszai A, Linke R and Kuwert T: Impact of 131I SPECT/spiral CT on nodal staging of differentiated thyroid carcinoma at the first radioablation. J Nucl Med 50: 18-23, 2009. PMID: 19091884. DOI: 10.2967/jnumed.108.052746

8 Pacini F, Molinaro E, Castagna MG Agate L, Elisei R, Ceccarelli C, Lippi F, Taddei D, Grasso L and Pinchera A: Recombinant human thyrotropin-stimulated serum thyroglobulin combined with neck ultrasonography has the highest sensitivity in monitoring differentiated thyroid carcinoma. J Clin Endocrinol Metab 88: 3668-3673, 2003. PMID: 12915653. DOI: 10.1210/ jc.2002-021925

9 Han JM, Kim WB, Yim JH, Kim WG, Kim TY, Ryu JS, Gong G, Sung TY, Yoon JH, Hong SJ, Kim EY and Shong YK: Longterm clinical outcome of differentiated thyroid cancer patients with undetectable stimulated thyroglobulin level one year after initial treatment. Thyroid 22: 784-790, 2012. PMID: 22780573. DOI: $10.1089 /$ thy.2011.0322.

10 Sherman SI, Tielens ET, Sostre S, Wharam MD Jr and Ladenson PW: Clinical utility of posttreatment radioiodine scans in the management of patients with thyroid carcinoma. J Clin Endocrinol Metab 78: 629-634, 1994. PMID: 8126134. DOI: 10.1210/jcem.78.3.8126134

11 Sherman SI: Thyroid carcinoma. Lancet 361(9356): 501-511, 2003. PMID: 12583960. DOI: 10.1016/s0140-6736(03)12488-9

12 Tharp K, Israel O, Hausmann J Bettman L, Martin WH, Daitzchman M, Sandler MP and Delbeke D: Impact of 131ISPECT/CT images obtained with an integrated system in the follow-up of patients with thyroid carcinoma. Eur J Nucl Med Mol Imaging 31: 1435-1442, 2004. PMID: 15221294. DOI: 10.1007/s00259-004-1565-2

13 Barwick TD, Dhawan RT and Lewington V: Role of SPECT/CT in differentiated thyroid cancer. Nucl Med Commun 33: 787-798, 2012. PMID: 22669053. DOI: 10.1097/MNM.0b013e3283550257

14 Avram AM: Radioiodine scintigraphy with SPECT/CT: an important diagnostic tool for thyroid cancer staging and risk stratification. J Nucl Med Technol 42: 170-180, 2014. PMID: 25190758. DOI: 10.2967/jnumed.111.10413

15 Wong KK, Zarzhevsky N, Cahill JM, Frey KA and Avram AM: Incremental value of diagnostic 131I SPECT/CT fusion imaging in the evaluation of differentiated thyroid carcinoma. AJR Am J Roentgenol 191: 1785-1794, 2008. PMID: 19020251. DOI: 10.2214/AJR.08.1218 
16 Yamamoto Y, Nishiyama Y, Monden T, Matsumura Y, Satoh K and Ohkawa M: Clinical usefulness of fusion of 131I SPECT and $\mathrm{CT}$ images in patients with differentiated thyroid carcinoma. J Nucl Med 44: 1905-1910, 2003. PMID: 14660715.

17 Kohlfuerst S, Igerc I, Lobnig M, Gallowitsch HJ, GomezSegovia I, Matschnig S, Mayr J, Mikosch P, Beheshti M and Lind P: Posttherapeutic (131) I SPECT-CT offers high diagnostic accuracy when the findings on conventional planar imaging are inconclusive and allows a tailored patient treatment regimen. Eur J Nucl Med Mol Imaging 36: 886-893, 2009. PMID: 19169681. DOI: $10.1007 / \mathrm{s} 00259-008-1044-2$

18 Aide N, Heutte N, Rame JP, Rousseau E, Loiseau C, HenryAmar $\mathrm{M}$ and Bardet $\mathrm{S}$ : Clinical relevance of single-photon emission computed tomography/computed tomography of the neck and thorax in postablation (131) I scintigraphy for thyroid cancer. J Clin Endocrinol Metab 94: 2075-2084, 2009. PMID: 19276233. DOI: $10.1210 /$ jc.2008-2313

19 Wakabayashi H, Nakajima K, Fukuoka M, Inaki A, Nakamura A, Kayano D and Kinuya S: Double-phase (131) I whole body scan and (131) I SPECT-CT images in patients with differentiated thyroid cancer: their effectiveness for accurate identification. Ann Nucl Med 25: 609-615, 2011. PMID: 21725630. DOI: $10.1007 / \mathrm{s} 12149-011-0513-\mathrm{Z}$

20 Xue YL, Qiu ZL, Song HJ and Luo QY: Value of 131 I SPECT/CT for the evaluation of differentiated thyroid cancer: a systematic review of the literature. Eur J Nucl Med Mol Imaging 40: 768778, 2013. PMID: 23242250. DOI: 10.1007/s00259-012-2310-x.

21 Zilioli V, Peli A, Panarotto MB Magri G, Alkraisheh A, Wiefels C, Rodella C and Giubbini R: Differentiated thyroid carcinoma: Incremental diagnostic value of 131I SPECT/CT over planar whole body scan after radioiodine therapy. Endocrine 56: 551559, 2017. PMID: 27709475. DOI: 10.1007/s12020-016-1086-3

22 Ciappuccini R, Heutte N, Trzepla G Rame JP, Vaur D, Aide N and Bardet S: Postablation 131I scintigraphy with neck and thorax SPECT-CT and stimulated serum thyroglobulin level predict the outcome of patients with differentiated thyroid cancer. Eur J Endocrinol 164: 961-969, 2011. PMID: 21471170 DOI: $10.1530 / E J E-11-0156$

23 Lee CH, Jung JH, Son SH, Hong CM, Jeong JH, Jeong SY, Lee SW, Lee J and Ahn BC: Risk factors for radioactive iodine-avid metastatic lymph nodes on post I-131 ablation SPECT/CT in low- or intermediate-risk groups of papillary thyroid cancer. PLoS One 13(8): e0202644, 2018. PMID: 30118516. DOI: 10.1371 /journal.pone .0202644
24 Haugen Br, Alexander EK, Bible KC, Doherty GM, Mandel SJ, Nikiforov YE, Pacini F, Randolph GW, Sawka AM, Schlumberger M, Schuff KG, Sherman SI, Sosa JA, Steward DL, Tuttle RM and Wartofsky L: 2015 American thyroid association management guidelines for adult patients with thyroid nodules and differentiated thyroid cancer: The American thyroid association guidelines task force on thyroid nodules and differentiated thyroid cancer. Thyroid 26: 1-133, 2016. PMID: 26462967. DOI: $10.1089 /$ thy 2015.0020

25 Oral A, Yazici B, Eraslan C and Burak Z: Unexpected false positive I-131 uptake in patients with differentiated thyroid carcinoma. Mol Imaging Radionuclide Ther 27: 99-106, 2018. PMID: 30317832. DOI: $10.4274 /$ mirt.37450

26 Choudhury PS and Gupta M: Differentiated thyroid cancer theranostics: radioiodine and beyond. Br J Radiol 91(1091): 20180136, 2018. PMID: 30260232. DOI: 10.1259/bjr.20180136

$27 \mathrm{Wu}$ YJ, Wu HS, Yen RF, Shen YY and Kao CH: Detecting metastatic neck lymph nodes in papillary thyroid carcinoma by $18 \mathrm{~F}-2$-deoxyglucose positron emission tomography and Tc-99m tetrofosmin single photon emission computed tomography. Anticancer Res 23: 2973-2976, 2003. PMID: 12926147.

$28 \mathrm{Wu}$ HS Huang WS, Liu YC, Yen RF, Shen YY and Kao CH: Comparison of FDG-PET and technetium-99m MIBI SPECT to detect metastatic cervical lymph nodes in well-differentiated thyroid carcinoma with elevated serum HTG but negative I-131 whole body scan. Anticancer Res 23: 4235-4238, 2003. PMID: 14666632.

29 Burlison JS, Hartshorne MF, Voda AM, Cocks FH and Fair JR: SPECT/CT localization of oral radioiodine activity: a retrospective study and in-vitro assessment. Nucl Med Commun 34: 1216-1222, 2013. PMID: 24128897. DOI: 10.1097/ MNM.0000000000000004

30 Ciappuccini R, Blanchard D, Rame JP, de Raucourt D, Babin E and Bardet S: Radioiodine sinus uptake related to mucosal thickening or aspergilloma: a case series of an unrecognized event well evidenced by SPECT/CT. Cancer Imaging 17: 2, 2017. PMID: 28086986. DOI: 10.1186/s40644-016-0105-1

31 Chudgar AV and Shah JC: Pictorial review of false-positive results on radioiodine scintigrams of patients with differentiated thyroid cancer. Radiographics 37: 298-315, 2017. PMID: 28076008. DOI: $10.1148 /$ rg.2017160074

Received July 8, 2019

Revised July 25, 2019

Accepted July 31, 2019 